\title{
Valbenazine and Deutetrabenazine as possible treatments for neuroleptic-induced supersensitivity psychosis and antipsychotic dependence
}

\author{
Richard Skaff*
}

Received 29 March 2018; Accepted 2 May 2018; First published online 21 November 2018

Dear Editor,

The article in the February 2018 issue of CNS Spectrums titled "Mechanism of action of vesicular monoamine transporter 2 (VMAT2) inhibitors in tardive dyskinesia"1 has thoroughly elucidated the mechanism of dysfunction in tardive dyskinesia (TD) and illustrated the latest treatments for TD with novel drugs like valbenazine and deutetrabenazine. However, the author did not address the bigger picture or the possibility of using these drugs off-label to treat other disorders with similar dysfunction such as supersensitivity psychosis.

In 2017 the FDA approved the first treatments for TD. The newly approved drugs are a highly selective vesicular monoamine transporter 2 inhibitors valbenazine (Ingrezza, Neurocrine Biosciences) ${ }^{2}$ and deutetrabenazine (Austedo, Teva). ${ }^{3}$

TD is thought to result from neostriatal dopaminergic receptor supersensitivity induced by protracted treatment with neuroleptics, or as Dr. Stahl describes it, as imbalance in "stop" and "go" signals from the motor striatum. ${ }^{1}$ Interestingly, in the 1970 s 2 physicians at McGill University, Chouinard and Jones, suggested that dopaminergic receptor supersensitvity also occurs in the mesolimbic region after chronic neuroleptic exposure, resulting in the development of what they called "supersensitivity psychosis," leading toward psychotic relapse by more than just the course of the normal illness. ${ }^{4}$ Furthermore, a 2012 study by Fallon, Dursun, and Deaken used a checklist of diagnostic criteria for supersensitivity psychosis and found it present in $39 \%$ of relapses. ${ }^{5}$ Relapse is a significant issue that plagues every patient. It has been estimated that for people with schizophrenia there is $40 \%$ relapse rate on medication in the first year following discharge from hospital; therefore identifying

\footnotetext{
* Address for correspondence: Richard Skaff, MA, MS, PsyD, PO Box 2782, Palos Verdes Peninsula, CA 90274, USA.

(Email: skaffrichar@aol.com)
}

the causes of relapse is critical, as it impacts the option of treatment. ${ }^{6}$ Moreover, this iatrogenic "supersensitivity psychosis" hypothesis leads us to ask the following questions: Is there a correlation between neostriatal dopaminergic receptor supersensitivity and mesolimbic DA postsynaptic supersensitivity leading to tardive psychosis? Could dopaminergic pathways become permanently dysfunctional and entrapped in a hyperactive state as a result of persistent use of antipsychotics? Could a treatment for TD be effective for supersensitivity psychosis as well?

More research is needed to answer these important and complicated questions. Furthermore, supersensitivity psychosis is an extremely important concept for today's prescriber due to the overuse of neuroleptics as a panacea in children and adults. This hypothesis should be revisited and honestly discussed in the psychiatric community regardless of the concerns about political correctness in regard to drugs and the pharmaceutical companies. Therefore, understanding the supersensitivity mechanism and its treatment is essential to provide our patients with better outcome.

In conclusion, the approval of the VMAT2 inhibitors valbenazine and deutetrabenazine for TD, combined with the high level of relapse triggered by long-term use of antipsychotics, as well as the apparent correlation between DA receptor supersensitivity in the mesolimbic and neostriatal regions, should motivate researchers to conduct a thorough study of valbenazine's and deutetrabenazine's efficacy (off-label) as single agents in the treatment of supersensitivity psychosis. By selectively reducing the ability of VMAT2 to load dopamine into synaptic vesicles, the drug reduces overall levels of available dopamine in the synaptic cleft, hypothetically mitigating the symptoms associated with dopamine hypersensitivity in the neostriatal and mesolimbic regions, thus targeting 2 birds with 1 effective stone. 


\section{Disclosures}

Richard Skaff has nothing to disclose.

\section{REFERENCES:}

1. Stahl S. Mechanism of action of vesicular monoamine transporter 2 (VMAT2) inhibitors in tardive dyskinesia: reducing dopamine leads to less "go" and more "stop" from the motor striatum for robust therapeutic effects. CNS Spectr. 2018; 23(1): 1-6.

2. Brauser D. FDA approves first treatment for tardive dyskinesia: valbenazine (Ingrezza). April 11, 2017. https://www.medscape. com/viewarticle/878495.
3. Brook M. FDA OKs deutetrabenazine (Austedo) for tardive dyskinesia. August 30, 2017. https://www.medscape.com/ viewarticle/885051.

4. Chouinard G, Jones BD. Neuroleptic-induced supersensitivity psychosis: clinical and pharmacologic characteristics. Am J Psychiatry. 1980; 137(1): 16-21.

5. Fallon P, Dursun S, Deakin B. Drug-induced supersensitivity psychosis revisited: characteristics of relapse in treatmentcompliant patients. Ther Adv Psychopharmacol. 2012; 2(1): 13-22.

6. Hogarty GE, Ulrich RF. The limitations of antipsychotic medication on schizophrenia relapse and adjustment and the contributions of psychosocial treatment. J Psychiatry Res. 1998; 32(3-4): 243-250. 